\title{
Konstruktion, Intuition: algorithmisch
}

\author{
Frieder Nake ${ }^{1}$
}

Angenommen: 15. Januar 2021 / Online publiziert: 12. Februar 2021

(c) Der/die Autor(en) 2021

\section{Zusammenfassung}

Konstruktion ist der starke Pfeiler aller Ingenieursarbeit, so auch der Arbeit des Programmierens. Konstruktives Handeln zieht jedoch explizit auch in Textgestaltung und Bildende Kunst des 20. Jahrhunderts ein. Intuition ist das Finden ohne Suchen, das künstlerisches Schaffen oft auszuzeichnen scheint. Sie steht aber gewiss auch bei manchem Programmiertrick Pate. Mit dem für informatisches Tun zentralen algorithmischen Denken werfen wir einen Blick zurück auf den Beginn ästhetischer Experimente mit Rechenmaschinen Ende der 1950er und Anfang der 1960er Jahre.

„Wir konstruieren und konstruieren und doch ist Intuition immer noch eine gute Sache," schreibt Paul Klee 1928 am Bauhaus [3]. Mit leisen Worten drückt er aus, wie er sich gefühlt haben mag im Kreise der in den 1920er-Jahren von rationalem Konstruieren begeistert erfassten Künstler und Künstlerinnen.

Der zitierte Satz Klees ist der erste eines kurzen Essays des Titels: „Exakte Versuche im Bereiche der Kunst“. Ohne Intuition mag man lange etwas tun, meint Paul Klee dort, systematisch mag man konstruieren. Exaktheit kann, gepaart mit Intuition, „beflügelt“ werden. Vieles können wir tun und weiter tun im Konstruieren. ,... und doch hat es,“ sagt Paul Klee, „eine Not: die Intuition ist trotzdem ganz nicht zu ersetzen." Man gelange nämlich ohne sie nicht zur „Totalisation“. Das Ganze, das Abschließende, die gelungene Gestalt, sie verlangen nach einem Weiteren, das zu benennen, Klee für heutiges Denken ein schweres, ein mittlerweile wohl verpöntes Gewicht einbringt: das Genie.

„Man war fleißig;“" schreibt er - ,aber Genie ist nicht Fleiß.“ Und kurz darauf: „Genie ist Genie, ist Begnadung, ist ohne Anfang und Ende" [3]. Wir werden uns mit der Romantik bescheiden müssen, die im Genie-Begriff schwingt. Klee war das, was er „Genie“ nennt, auch wenn das Wort uns fremd geworden sein mag, aus der Zeit gefallen. Und doch wird das, was Wort und Begriff einst auszudrücken in der Lage waren, auch unter uns, die wir verloren sind im Rationalen, noch immer existieren und unbemerkt auch wirken in dieser Zeit des Algorithmischen.

\footnotetext{
Frieder Nake

nake@uni-bremen.de

1 Universität Bremen, Bremen, Deutschland
}

Kann Kunst geschehen, kann einer künstlerisch arbeiten, ohne dass beide zusammen anklängen, Konstruktion und Intuition? Liegt Künstlerisches nicht geradezu mitten in diesem scheinbar widersprüchlichen Klang? Kaum anders scheint es mir denkbar. Und verhält es sich mit dem Algorithmischen heute anders als damals mit dem Konstruktiven? Darum soll es uns gehen.

„Es gibt gar keine Elektronengehirne.“ Mit dieser schlichten Feststellung beginnen Rolf Lohberg und Theo Lutz ihr 1963 vorgelegtes Buch, „Was denkt sich ein Elektronengehirn?" [5]. Lesen wir eine solche Zeile heute, nahezu 60 Jahre später, so schmunzeln wir wohl, schütteln vielleicht mitleidig seufzend den Kopf oder greifen uns amüsiert an die Stirn. Himmel, geht es mir durch den Kopf, wie blöd müssen die doch gewesen sein, so auch nur zu fragen (Abb. 1).

Theo Lutz war keine 10 Jahre älter als ich. Aber im Jahr 1963, als ich damit anfing, per Algorithmus dem Computer Zeichnungen zu entlocken, wäre mir ein solch albernes Wort wie das vom Elektronengehirn und seinem Denken nicht über die Lippen geraten. Lag das Interesse solch eines Titels nur in der provokanten Frage, in der Aufmerksamkeit, die er erzeugte? War das schon alles?

\section{Algorithmisch denken}

Denken wir zurück! Zu jenem Zeitpunkt, also 1963, als man offenbar noch ungestraft vom „Denken elektronischer Gehirne" sprechen konnte, ist die Programmiersprache Algol 60 bereits 3 Jahre alt (eigentlich sogar ein bisschen älter) [2, 8]. Entwicklung und Verständnis der Programmiersprachen erreichen mit Algol einen Höhepunkt. Wer an einer 


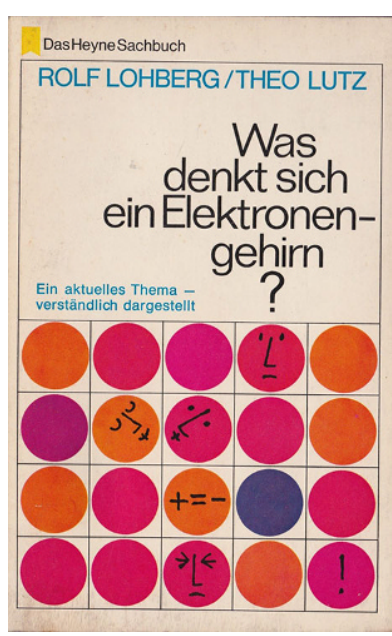

Abb. 1 Titel des Buches von Lohberg und Lutz [5]

westdeutschen Universität etwas auf sich hält und zu denen zählt, die die Zukunft anfassen, kennt jene Sprache Algol und muss sie eigentlich auch beherrschen. Unter den heutigen Lesern des Informatik Spektrum (das im August 1978 erstmals erschien) - so darf ich annehmen - wird es noch immer eine stattliche Gruppe von Menschen geben, die einst Texte in Algol sicher und elegant formulieren konnten und die auch heute noch wissen, wofür der Name dieser künstlich geschaffenen Sprache steht. Sie mögen deswegen wohl auch heute noch zu solchem in der Lage sein, weil Algol hinlänglich ähnlich zu dem war, was wir von einer „Sprache“ erwarten. Einmal gelernt, bleibt eine Sprache mit uns; ein wenig mag unsere Kenntnis sich abschwächen, wenn wir die Sprache nicht mehr gebrauchen. Doch sie bleibt mit uns.

Ihr Name - „Algol“ - aber enthüllt uns, dass es sich eben doch nicht wirklich um eine der Sprachen von der Art handelt, die mit uns sind und wachsen. Sie ist ein Artefakt, eine Konstruktion, nützlich für gewisse Aufgaben, unnütz für alles andere. Diese Konstruktion nun besitzt einen einzigen Zweck, den nämlich, Algorithmen zu formulieren. Deswegen heißt und ist sie eine „Algorithmic Language“, eine „algorithmische Sprache“. Ihr spezifischer Name ist die Abkürzung ihrer Genre-Bezeichnung zu nur einem Wort, eben „Algol“. Speziell haben wir es zu tun mit der Sprache Algol 60, so genannt, weil sie im Jahre 1960 öffentlich gemacht wurde. Das schon allein, das „Öffentlich-Machen“, sagt uns: Sprache so recht eigentlich kann das nicht sein. Wir sind, das wird deutlich, tief in der Welt der Künstlichkeit.

(Es mag, in Klammern sei es gesagt, als ein wenig befremdlich erscheinen, dass mit Algol 60 zwar eine große Woge hin zur Informatik als neuer akademischer Disziplin angestoßen wurde, dass es auch noch den ambitionierten und gescheiterten Versuch der zweischichtigen Sprache Algol 68 gab, dass aber außer der Technik, wie man die Syntax einer Programmiersprache ordentlich definiert, nahe- zu nichts mehr uns heute kümmert aus jener versunkenen Welt.)

Algorithmen also waren die Gegenstände, mit denen Algol es zu tun hatte. Das hohe Ziel der Gruppe von Autoren, die diese Sprache schufen, war es, dass von nun an Algorithmen stets und überall in Algol formuliert werden sollten. Universell sollte diese Sprache werden. Ein strenger Wille zum Einheitlich-Allumfassenden umhüllte ihre Konstruktion. Sie war gut, also brauchte es keine andere daneben.

Die Association for Computing Machinery (ACM) hatte bereits im Februar 1960 damit begonnen, in einer eigenen Sektion ihrer Zeitschrift Communications of the ACM (CACM) laufend Algorithmen zu veröffentlichen. Ein Schatz von sorgfältig formulierten und geprüften Algorithmen entstand, damals noch in der Mehrzahl für mathematische Aufgaben gedacht. Aus Tat sollte allgemeine Gültigkeit entstehen. Denn alle diese Algorithmen in den CACM mussten nun in Algol präsentiert werden. Bald genug kam eine Diskussion darüber auf, wie das denn mit Fortran sei als einer alternativen Publikations-Sprache für Algorithmen [8]. Schließlich war das die Sprache der Praktiker und sie war weiter verbreitet und bekannter als das europalastige Algol 60.

Die frühen 1960er-Jahre markieren einen Höhepunkt der Algorithmik: der Entwicklung, Veröffentlichung, Verbesserung von Algorithmen. Immer lauert hinter ihnen die Praxis der Programmierung. Der Gegenstand dieser Praxis ist das Programm, mit ihm auch die Programmiersprache und schließlich der Computer. Algorithmisches Denken aber, ein Denken in und hin zu Algorithmen, ist unabhängig von den Engen, Höhen und Tiefen des Programmierens, und es muss auch unabhängig sein von ihnen. Algorithmisches Denken ist zunächst kein Denken zum Computer hin, sondern, zugespitzt gesagt, vom Menschen weg und damit aber immer zunächst bei ihm.

Ein Problem möchte der Mensch nun so lösen, dass eine Vorschrift entsteht, die präzise und eindeutig in jedem der Schritte einer Folge von Operationen ist, die selbst stets effektiv enden müssen. Das bedeutet, dass am Ende Operationen stehen müssen, über deren Bedeutung (und damit Ausführung) keinerlei Zweifel bestehen darf. Über einem Grundstock von vorgegebenen und fraglosen Operationen baut ein Algorithmus eine komplexe Entscheidungsstruktur auf, die die Welt der effektiven Operationen nicht verlässt, sondern sie auf komplexe Weise ständig erweitert.

Wenn es dann aber schließlich dazu kommen soll, dass ein Computer jene Instanz ist, die die effektiven Operationen ausführen soll, dann muss die algorithmische Beschreibung des Lösungsgangs in einer dem Computer zugänglichen Notation erfolgen - und das ist eine Programmiersprache. Der Algorithmus, für den Menschen geschrieben, wird zum Programm, der Maschine angepasst. 
Dass eine solche Darstellung die Verhältnisse der Praxis gegenüber stark vereinfacht, ist offensichtlich. Doch wie könnt' es anders sein? Und, in einem alten, schönen Lehrbuch zurückblätternd, lesen wir: „An algorithm must be seen to be believed" [4, p. 4]. In seiner grandios induktiven Schreibweise scheut Donald Knuth sich nicht, von Algorithmen als Gegenständen des Glaubens zu sprechen. Einen irdischen Glauben spricht er an, der dann nämlich möglich wird, wenn wir den Algorithmus sehen können. Damit wird er in Gänze zu einem endlichen Gegenstand. Das unterscheidet die Informatik von der Mathematik.

Niemand hat je die Menge $\mathbb{R}$ der reellen Zahlen gesehen, ja, noch nicht einmal die der ganzen Zahlen, $\mathbb{Z}$. Wir können ihnen Namen geben, diesen ansonsten nur vorgestellten Gebilden. Die mathematische Konstruktion der unendlichen Menge $\mathbb{Z}$ ist etwas radikal anderes als das, was in der Informatik der oft int genannte "Typ“ ist - die endliche Menge jener ganzen Zahlen, die der Datentyp integer in grandioser Verknappung von $\mathbb{Z}$ enthält. Mathematik befasst sich mit Unendlichkeiten, Informatik hingegen mit Endlichkeiten. Vieles holt die Informatik sich aus der Mathematik, das meiste aber lässt sie dort. Etliches schafft die Informatik dann, das große praktische Wirkung erzielt. Geistige Vorstellung aber bleibt in der Mathematik.

Sehen müssen wir den Algorithmus, sagt Don Knuth, um ihm glauben zu können. Aus seinem Munde gewinnt so etwas großes Gewicht, ist er doch Christ. Mit guten Gründen könnten wir sagen, Algorithmen seien rekursive Funktionen. Auch könnten wir davon sprechen, dass sie von Turing-Maschinen ausführbar sein müssen. Solch eine Auffassung schlägt sich nieder in gut bekannten Anforderungen, denen eine Beschreibung (ein Text also) genügen müsse, um „Algorithmus“ genannt zu werden: die schon erwähnten Attribute der Endlichkeit, Präzision, Eindeutigkeit und Effektivität, zu denen noch genaue Festlegungen der Datentypen hinzukommen müssen, und oft auch noch die Endlichkeit jeder einzelnen Ausführung. Mit ein wenig zusätzlicher Schärfe versehen können wir durchaus sagen: „So zu denken, wie ein Computer dächte, wenn er es könnte - das ist algorithmisch zu denken."

\section{Algorithmische Texte}

Mit der algorithmischen Sprache Algol, die - nach ihrem Selbstverständnis - recht eigentlich keine Programmier-, sondern eine algorithmische Sprache ist, erreichen die frühen 1960er-Jahre einen Höhepunkt des scharfen, präzisen, eleganten, ja, tatsächlich auch ästhetischen Denkens. Fast notwendigerweise drängt das zu einer eigenen Disziplin. Mit der Computer Science und der Informatik entsteht sie. Das Wort „Informatik“ wird im Juli 1968 in einer Rede des Wissenschaftsministers der Bundesregierung für ein neu zu
Nicht jeder Blick ist nah. Kein Dorf ist spaet. Ein Schloss ist frei und jeder Bauer ist fern. Jeder Fremde ist fern. Fin Tag ist spaet. Jedes Haus ist dunkel. Ein Auge ist tief.

Abb. 2 Algorithmisch erzeugter Text, Theo Lutz mit Rul Gunzenhäuser [6]

gründendes Studienfach vorgeschlagen. Die Industrie hatte einen Mangel an Fachpersonal in der Datenverarbeitung beklagt. Die Politik beschloss zu investieren.

Dem schmalen Buch darüber, was Elektronengehirne nicht nur denken, sondern gar sich denken mögen, das ich eingangs erwähnt hatte, war in den 1960er-Jahren durchaus eine stattliche Leserschaft vergönnt; es erlebte in seiner Zeit 36 Auflagen und wurde in mehrere Sprachen übersetzt. Das Werk findet sich auf meinem Bücherregal, weil ich damals nachlesen wollte, welche Art von Denken sich hinter der arg provokanten Titel-Frage verbergen mochte, die im Untertitel bereits zurückgenommen wurde: Gemeint war das Werk als eine ,verständliche Einführung in die Arbeitsweise der Elektronenrechner“. Das Denken dieser merkwürdigen Gehirne wurde im Geschwindschritt auf das Rechnen einer Maschine reduziert. Das Arbeiten dieser Maschine sollte größeren Kreisen der interessierten Bevölkerung nähergebracht werden.

Einer der beiden Autoren, Rolf Lohberg, war Journalist. Er schrieb populär über Technisches. Sein Mitautor, Theo Lutz, war in der Branche der Datenverarbeitung in Stuttgart tätig. Er wurde bekannt durch weltweit erste computergenerierte Texte. Sein Freund, Rul Gunzenhäuser, half ihm bei der Programmierung. Als Lutz die Ergebnisse dem Stuttgarter Philosophen Max Bense zeigte, reagierte dieser begeistert und gab ihm Gelegenheit zur Publikation in der literarischen Zeitschrift augenblick. Einige Beispielzeilen aus einem 1959 algorithmisch generierten Text zeigt Abb. 2.

Viele Leser solcher Zeilen werden in der Lage sein, mühelos einen Algorithmus zu notieren, der in der Lage ist, diese und ähnliche Zeilen zu generieren. Vor diese Aufgabe gestellt, würden sie damit beginnen, in den gegebenen Textzeilen nach Mustern und Regeln zu suchen. In unserem Fall ist diese Aufgabe trivial. Denn wir bemerken sofort, dass jede Zeile zwei sehr einfache Aussagesätze enthält. Jeder der Sätze folgt dem gleichen Schema. Ein Substantiv wird mit einem Adjektiv durch das Verb „ist“" gekoppelt. Dem Substantiv wird eine Quantifizierung vorangestellt (wie ein, kein, jeder). Die beiden Sätze werden schlicht hintereinander gesetzt oder mit ,und“ gepaart (vermutlich sind weitere Möglichkeiten erlaubt, wie etwa „oder“, „,aber“, ,jedoch“). Jeder Satz verlangt nach drei zufälligen Wort-Wahlen: das Substantiv, das Adjektiv und die Quantifizierung. Dazu kommt die Wahl der Satzverbindung, auch sie zufallsbestimmt. Sammlungen von Wörtern müssen vorgegeben werden, aus denen ausgewählt werden 
Abb. 3 Theo Lutz (a) und Rul Gunzenhäuser (b)
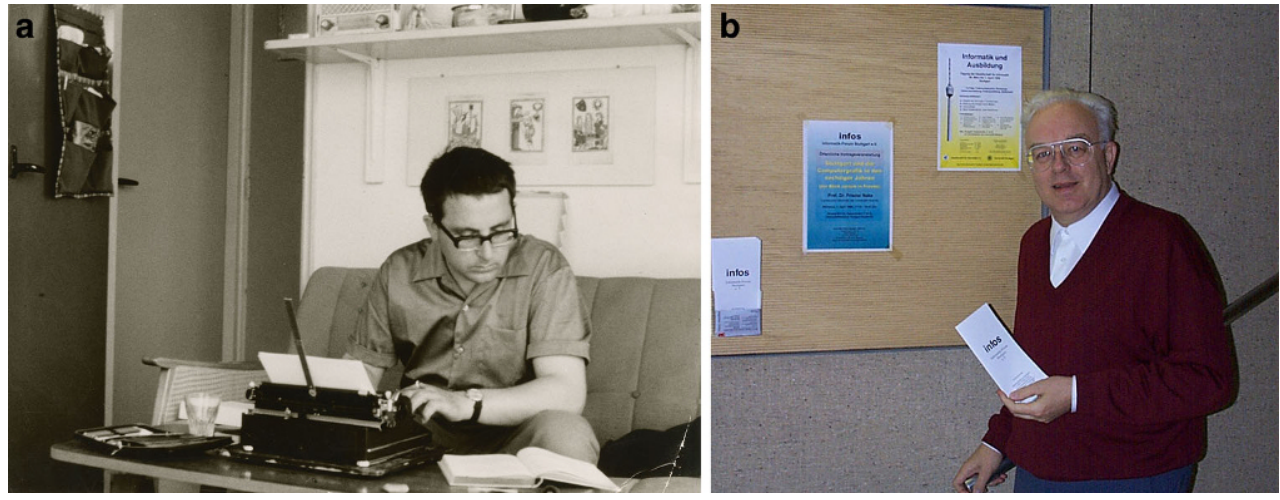

kann - und fertig ist der Textgenerator, also Algorithmus, der solche Texte fabrizieren kann (Abb. 3).

Das oben in gewöhnlicher Sprache beschriebene Erzeugungsschema mag uns als so simpel erscheinen, dass wir es kaum als ,algorithmisch“ qualifizieren würden. Das jedoch wäre falsch. Denn es bleibt eine nicht hintergehbare Notwendigkeit auch für komplexere algorithmische Handlungsanweisungen, dass sie auf vorgegebene, effektiv durchführbare Elementaroperationen führen. Diese Operationen werden als effektiv durchführbar vorausgesetzt, was nichts anderes bedeutet, als dass letzten Endes eine mechanische (oder andersartige maschinelle) Ausführung vorliegt.

Eine wichtige Eigenschaft allerdings muss der Algorithmus der Lutz'schen Texte aufweisen, damit das geschehen kann, was zu jener Zeit für einiges Aufsehen sorgte: Wie nämlich kommt es zur Bestimmung des Substantivs und des Adjektivs in jedem der kurzen Sätze? Und was ist der Grund für das Wort „stochastisch“ im Aufsatz von Lutz? Es ist genau das, was ,stochastisch“ meint: Die Wörter werden aus ihren spezifischen Vorratsmengen (aus ihrem jeweiligen Repertoire) zufällig ausgewählt. Das geschieht über Wahrscheinlichkeitsverteilungen und Pseudo-Zufallszahlen. Die überraschenden, erheiternden oder befremdlichen Wirkungen, die derartige Texte erzeugen konnten, hatten einerseits durchaus Vorbilder in der modernen Poesie. Andererseits aber waren sie sehr nüchtern dem Wirken algorithmischer Zufallszahlengeneratoren zu verdanken.

Wenn diese Art des „Dichtens als Berechnen“ 1959 auch zu Wellen kultureller Aufregung führte, so kann das kaum der Schlichtheit der Algorithmisierung angelastet werden. Es wird vielmehr der Affront gewesen sein, dass auf algorithmische Weise, also vom Computer mit Programm, Texte erzeugt wurden, die mit einer gewissen Ernsthaftigkeit als ästhetische Objekte in die Welt entlassen wurden. Zur selben Zeit wurden schon längst geistige Tätigkeiten von ganz anderem Kaliber algorithmisch beschrieben. Wo immer solches geschieht, geschieht eine Maschinisierung von Kopfarbeit [7]. Sie ist das, was Reaktionen des Schreckens, aber auch der Begeisterung bewirken kann. Was eben noch dem Menschen mit Geist vorbehalten schien zu tun, taucht nun an der Peripherie eines Computers mit Programm auf.

Jede Algorithmisierung ist Form, in der Maschinisierung einer konkreten geistigen Arbeit erscheint; oder, etwas anders gesagt: Jede Algorithmisierung kann in einer Maschinisierung geistiger Arbeit münden. Längst ist dieser weltweit stattfindende gesellschaftliche Prozess breit akzeptiert. Er ist alltäglich und somit selbstverständlich geworden. Fast können wir uns nicht mehr vorstellen, dass gesellschaftliches Leben auch ohne solche Maschinisierung noch gelingen könnte. Wird dabei die Welt der Künste getroffen, so kann das auch heute noch gelegentlich zu Unverständnis, Abwehr oder Verachtung führen, gute $60 \mathrm{Jahre}$, nachdem in den 1950er- und 1960er-Jahren Musik, Grafik und eben auch Text algorithmisch erzeugt wurden.

\section{Algorithmische Kunst}

„Rechnen heißt, aus gegebenen Angaben nach einer Vorschrift neue Angaben zu bilden“ [9]. Das hielt Konrad Zuse nach eigener Angabe als seinen Versuch fest, den Begriff des Rechnens zu beschreiben, als er „mit seinen Arbeiten begann“. „Seine Arbeiten“- das war, was ihn zur Konstruktion einer automatischen, durch ein Programm gesteuerten Rechenmaschine führte. Diese Arbeiten begann der Bauingenieur nach seiner Diplomprüfung (wohl 1936).

Seine Arbeiten waren erfolgreich. Im Jahre 1941 konnte er in der Wohnstube seiner Eltern einem kleinen Kreis von Menschen eine Rechenmaschine vorführen, die unter dem Namen ,Z3“ als erster Computer anerkannt ist. Der Welt blieb das verborgen. Es herrschte Krieg.

Konrad Zuse steuerte noch in den letzten Jahren seiner Zugehörigkeit zur Zuse KG in Bad Hersfeld der Welt des Computing eine weitere feine Konstruktion bei: eine frühe, vom Computer gesteuerte Zeichenmaschine, den Zuse Z64 Graphomat. Zu jener Zeit war das die Zeichenmaschine der höchsten Genauigkeit. Sie steuerte Punkte mit einer Präzision von $1 / 16 \mathrm{~mm}$ an (Abb. 4). 


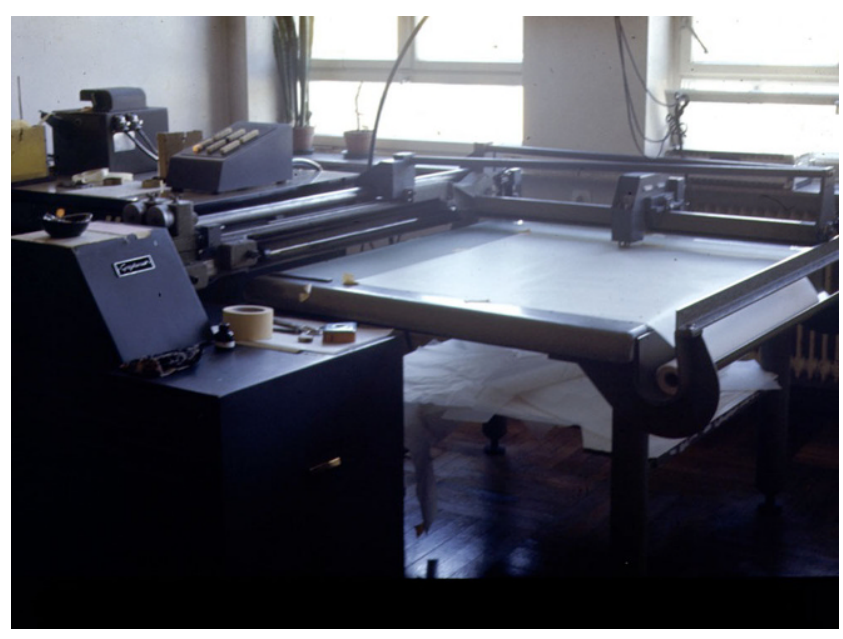

Abb. 4 Zuse Z64 Graphomat im Rechenzentrum TH Stuttgart

Das Rechenzentrum der Universität Stuttgart (die damals noch eine Technische Hochschule war) kaufte eines der ersten Exemplare des Graphomat. Sie erhielt jedoch von der Zuse KG für den Computer, mit dem der Stuttgarter Graphomat zusammenarbeiten sollte, keine Software. Denn der Stuttgarter Rechner war keine Zuse-Maschine. Ich war studentische Hilfskraft im Rechenzentrum und wurde wunderbarerweise mit der Aufgabe betraut, die notwendige Software zu entwickeln. Die Aufgabe entpuppte sich als der Glücksfall meines Lebens. Denn nun stand ich erstens vor der Aufgabe, mir zu überlegen, wie man die Tätigkeit des Zeichnens algorithmisch fasst. Und zweitens musste ich das allein mit Papier und Bleistift tun, ohne das beliebte Testen auf der Maschine. Denn der Stuttgarter Graphomat sollte erst im nachfolgenden Jahr geliefert werden (1964).

Die Leserin und auch der Leser mögen sich fragen, warum ich eine so nebensächliche Geschichte erzähle. Erwähnt habe ich diese Sache nur deswegen, weil ich zu einem für die Grafische Datenverarbeitung sehr frühen Zeitpunkt (wir sind 5 Jahre von der Informatik entfernt) begreifen durfte: Es wird keine gekrümmten Kurven mehr geben, wenn auf algorithmische Weise gezeichnet werden wird! Nur Polygone noch bevölkern die Welt algorithmischen Zeichnens. Das einzige Zugeständnis an die Welt der Kurven mit Krümmungen $\kappa \neq 0$ war, dass die approximierenden Polygone sehr viele und sehr kurze Kanten haben durften. Dass diese Polygone immer noch für das bloße Auge recht manierlich an Kreisbögen erinnernde Linien zustande bringen konnten, war der erwähnten Genauigkeit geschuldet. War das nun gut oder nicht gut für Künstlerisches?

Auf Graphomaten entstanden jedenfalls jene Zeichnungen, die Georg Nees im Februar 1965 weltweit erstmals in der Studiengalerie der TH Stuttgart, und die ich im November 1965 in der Galerie Wendelin Niedlich in Stuttgart ausstellen konnten. Im April des Jahres zeigte die Howard
Wise Gallery in New York ähnlich anmutende Zeichnungen von A. Michael Noll. Aus Amerika kam dann das Wort Computer Art. Nees und Max Bense verwendeten die Bezeichnung Generative Grafik. Heute hören wir immer noch und wieder von Computerkunst, vielleicht ein wenig klüger: Digitale Kunst. Doch richtig trifft erst Algorithmische Kunst.

Denn algorithmisch muss denken, wer sich an mathematische Verfahren und Methoden heranmachen will, deren Linien und Flächen als farbige Tuschen auf Papier die Kunstgeschichte herausfordern können. Das algorithmische Prinzip brach in das Zeichnen und Malen ein, indem aus Entfernung vom Zeichentisch das gedacht wurde, was bisher die geschickte Hand unmittelbar gezeichnet oder gemalt hatte. Dies aber ist das Prinzip des Algorithmischen: Die Distanz zum schließlich erstrebten materiellen Gegenstand ist geradezu grausam notwendig, um zu jener Freiheit des Gedankens zu gelangen, in der noch nichts materiell geschieht, in der der Gedanke schrankenlos ist, und wo er dennoch auf Wahrnehmbares abhebt, das nach materieller Existenz verlangt.

Der Riss zwischen Denken und Zeichnen, zwischen geistiger Vorstellung und materieller Tat, durch den Algorithmus überbrückt, ist das, was hier geschah. Ein ungeheurer Sprung des Geistes musste stattfinden, um algorithmisch das Sichtbare zu erfassen. In der Kunst war dafür vieles vorbereitet. Und die frühen Zeichnungen sind wunderbar brav. Es konnte nicht anders sein (Abb. 5).

\section{Konstruktion und Intuition}

Wie es war, wie ich vorgegangen sein mag, als ich zum ersten Mal vor der Aufgabe saß, den größten gemeinsamen Teiler zweier ganzer Zahlen zu bestimmen, weiß ich leider nicht mehr. Zu lange ist das her. Später habe ich dann, wie viele andere auch, im Kurs Algorithmen und Datenstrukturen, wie er für unser 1. Semester in Informatik hieß, flott auf die schöne rekursive Formulierung gezielt, mehr oder minder so:

if $(\mathrm{a}==\mathrm{b})\{\mathrm{t}=\mathrm{a} ;\}$

else if $(\mathrm{a}<\mathrm{b})\{\mathrm{t}=\operatorname{ggT}(\mathrm{b}, \mathrm{a}) ;\}$

else $\{\mathrm{t}=\operatorname{ggT}(\mathrm{a}-\mathrm{b}, \mathrm{b}) ;\}$

return $(\mathrm{t})$

Das kann einer leicht verstehen, mag man behaupten. Dabei unterstellen wir jedoch, längst sei klar, dass zwei ganze Zahlen entweder gleich sind, oder dass eine die gröBere der beiden ist. Und auch die 1. Zeile des algorithmischen Schemas ist sofort einsichtig: Sollten beide Zahlen gleich sein, dann ist ihr gemeinsamer Wert der gesuchte Teiler. So zu organisieren (2. Zeile), dass im Fall der Un- 
b
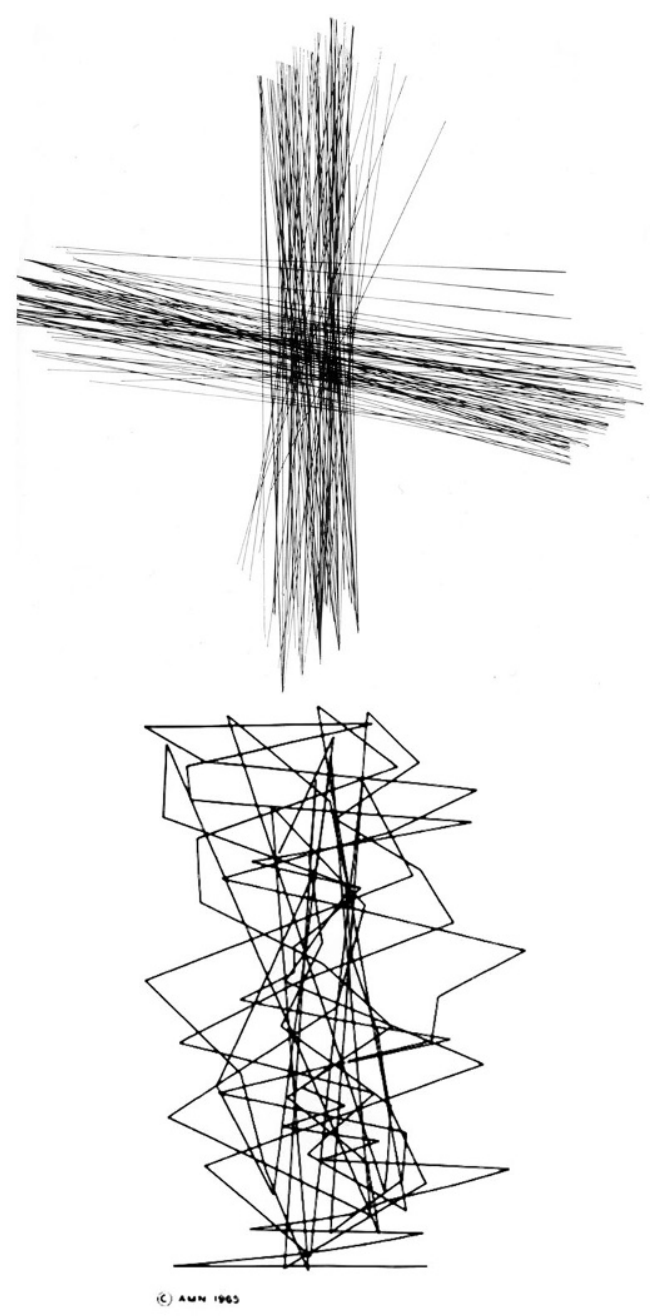

C

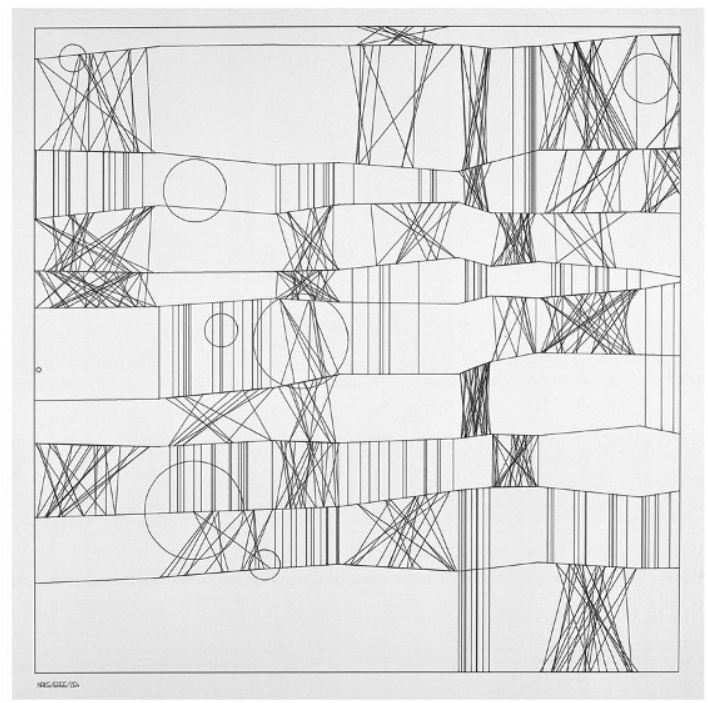

Abb. 5 Drei frühe algorithmische Bilder von 1965. Georg Nees: Andreaskreuz (a), A. Michael Noll: Gaussian Quadratic (b), Frieder Nake: $12 / 7 / 65$ Nr. $(\mathbf{c})$ gleichheit die größere der beiden an 1. Stelle stehen soll, leuchtet auch ein. Jedoch die 3. Zeile, in der es nun auf das Ziel zugeht, muss bewiesen werden. Das ist nicht schwer, aber mathematisches Denken muss helfen. Ohne das geht es nicht.

Was ist hier intuitiv? Das Vermögen der Studierenden, den relativ einfachen Gedanken zu folgen, die gedacht werden müssen, um diese rekursive Formulierung zu verstehen, vielleicht. Gewöhnung an Derartiges wird nicht viel an notwendiger Intuition übriglassen. Das Beispiel taugt ja auch viel mehr dazu, an einem einfachen Fall Rekursion zu begreifen als die Kunst des sparsamen Denkens. Nie ein rechnerisches Problem zu Ende zu formulieren, sondern es nach dem Prinzip maximaler Ästhetik auf sich selbst zurückzuführen und es nur im trivialen Fall explizit zu lösen (die 1. Zeile im Beispiel).

Was es hier zu konstruieren gilt, ist trivial. Die Wucht der Rückführung einer (einfachen) Berechnung auf sich selbst wird hier erreicht durch geschickte Reduktion der Parameterwerte in Richtung auf den trivialen Fall. Es ist, als hätte Euklid an die Informatik-Adepten gedacht, die Hunderte von Jahren nach ihm sich mit diesen Teilern herumschlagen müssten, obwohl sie daran kein Interesse haben (und Euklid hat das auch mehr als eine Frage des gemeinsamen Maßes interessiert als eine Frage der Zahlentheorie).

Wenn wir nach Algorithmischem in den 3 Grafiken der Abb. 5 fragen, sieht es gewiss schon anders aus. Die eine oder andere Leserin mag z.B. bemerken, dass die 3 . der Grafiken (Nake) in ihrer oberen rechten Ecke einen Programmierfehler enthält (ich möchte ihn nicht enthüllen, sondern als Rätsel bestehen lassen). Er wird dort 2-mal manifest, noch kein Mensch hat mich darauf angesprochen, und ich sehe ihn auch nur, weil ich von ihm weiß. Intuitiv wird er nicht auffällig. Haben wir ihn zunächst einmal nur als Phänomen entdeckt, so können wir ihn auch sogleich erklären und korrigieren. Aber Vorsicht! Wir müssen dazu mehr noch über die Art und Weise wissen oder vermuten, wie das Bild Schritt für Schritt, also algorithmisch, entsteht.

Diese Zeichnung aus dem Juli 1965 war in Maschinencode (ich vermeide das Wort „Sprache“) programmiert worden. Solchem Code fehlte jede Eleganz. Der Computer be$\mathrm{sa}$ einen zu kleinen Speicher. Deswegen musste, sobald eine Entscheidung getroffen war, die zur Bewegung des Zeichenstiftes führen sollte, der ihr entsprechende Code ausgegeben werden. In den meisten Fällen war er damit aus dem Speicher verschwunden. Die Komplexität der Zeichnung verlangte jedoch, einige lokale Elemente zu bewahren, um über sie etwas mehr Kohärenz des Ganzen zu bewahren. Die maschinellen Gegebenheiten verlangten derartige Gedankengänge und begrenzten manchen Gestaltungswunsch.

Jede Konstruktion verlangt nach einer gehörigen Aufmerksamkeit dem Ganzen gegenüber, zu dem sie beiträgt. Die Operationen der rechnenden Maschine hingegen sind 


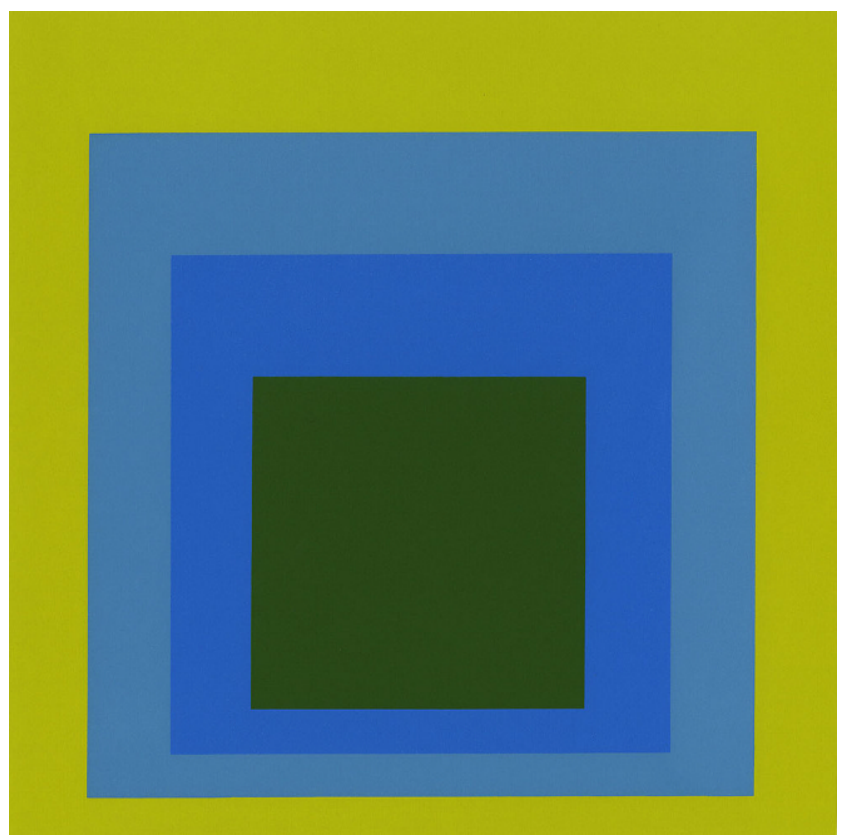

Abb. 6 Josef Albers: Homage to the Square. Soft Spoken, 1969

stets lokaler Natur. Nur in kluger Speicherung wichtiger Merkmale lässt sich in der Welt algorithmischer Zeichnung ihr entstehendes Ganzes als Orientierung für lokale Operationen bewahren. Zu Zeiten kleiner Speicher war das algorithmisch eine hohe Herausforderung. Das Auge des Künstlers wie auch der Künstlerin richtet den Blick stets aufs Ganze. Wie wäre in der algorithmischen Kunst dieses Auge zu simulieren?

Die frühen ästhetischen Experimente algorithmischer Kunst standen blind vor dieser Frage. Wie hat der knappe Speicher konstruktive Versuche beeinflusst, die 1965 Inspiration aus dem Betrachten von Zeichnungen von Paul Klee suchten? Schwer zu sagen. Doch meine Vermutung ist: Das Denken hin auf den Maschinencode muss stark gelitten haben unter der Enge der Sprache und des Speichers. Eine höhere Programmiersprache hätte zu anderer Konstruktion geführt. Denn die Konstruktion ist hier als ein Geflecht von einfachen effektiven Operationen zu organisieren, die es in einem Entscheidungsnetzwerk zusammenzuführen gilt.

\section{Intuition und Zufall}

Eine kurze Bemerkung darf ich mir noch erlauben zur Intuition, wenn es sie nicht mehr gibt, weil alles algorithmisch vonstattengehen muss. Wir wissen gar nicht so schlecht, was sie sei, die Intuition. Scharf und genau sagen aber können wir es nicht. Mit den wichtigen Begriffen unserer Wirklichkeit ist das oft so. Der junge Mensch in der Mitte der 1960er-Jahre sagte deswegen frech: Intuition? Das ist nichts als kluger Umgang mit Wahrscheinlichkeiten. Heute weiß jeder Mensch, dass es stets eine Random-Funktion gibt. Das war nicht immer so, man programmierte sich seine eigenen Folgen von Zufallszahlen. Viel besser aber noch: Kannte man Wahrscheinlichkeitstheorie, dann konnte man ganz anders - gezielt, differenziert, geschachtelt, betonend - mit dem Zufall umgehen. Das war eine Lust!

Indem ich in einer Zeichnung die Koordinaten jedes ihrer Punkte, jeden Radius eines Kreises, jeden Winkel zwischen 2 Geraden als Zufallsvariable modellierte und dies alles verschiedenen Wahrscheinlichkeitsverteilungen unterwarf, konnte ich so tun, als hätte ich keine Ahnung von der entstehenden Grafik und war dennoch die oberste Kontrollinstanz. Die algorithmische Kunst ist bis heute ein Fest, das an Algorithmen gebunden ist, die nichts als Wahrscheinliches sich zu ereignen gestatten. Das aber wäre eine weitere Geschichte.

Ich darf eine Kleinigkeit aus der Kunstgeschichte erzählen zum Abschluss. Vielen werden Bilder der großen Serie Homage to the Square von Josef Albers bekannt sein. Seit 1950 malte er sie, 25 Jahre lang, Hunderte. Das Form-Problem, die Konstruktion also, hatte er sich ein für alle Male vom Hals geschaffen. Vier Quadrate schachtelte er in einander, stets nach gleichen Proportionen, nach unten drängend. Jedes der sich überdeckenden Quadrate färbte er. Die Wahl der Farben war das Ergebnis seiner Intuition. Er sprach von den Bildern als der „Interaction of color“ ([1]; Abb. 6).

Funding Open Access funding enabled and organized by Projekt DEAL.

Open Access Dieser Artikel wird unter der Creative Commons Namensnennung 4.0 International Lizenz veröffentlicht, welche die Nutzung, Vervielfältigung, Bearbeitung, Verbreitung und Wiedergabe in jeglichem Medium und Format erlaubt, sofern Sie den/die ursprünglichen Autor(en) und die Quelle ordnungsgemäß nennen, einen Link zur Creative Commons Lizenz beifügen und angeben, ob Änderungen vorgenommen wurden.

Die in diesem Artikel enthaltenen Bilder und sonstiges Drittmaterial unterliegen ebenfalls der genannten Creative Commons Lizenz, sofern sich aus der Abbildungslegende nichts anderes ergibt. Sofern das betreffende Material nicht unter der genannten Creative Commons Lizenz steht und die betreffende Handlung nicht nach gesetzlichen Vorschriften erlaubt ist, ist für die oben aufgeführten Weiterverwendungen des Materials die Einwilligung des jeweiligen Rechteinhabers einzuholen.

Weitere Details zur Lizenz entnehmen Sie bitte der Lizenzinformation auf http://creativecommons.org/licenses/by/4.0/deed.de.

\section{Literatur}

1. Albers J (1975) Interaction of color. Yale University Press, New Haven, London

2. Backus JW et al (1963) Revised report on the algorithmic language ALGOL 60. Comput J 5(4):349-367

3. Klee P (1976) exacte versuche im bereich der kunst. In: Geelhaar Ch (Hrsg) Klee, P.: Schriften, Reflexionen und Aufsätze. DuMont, Köln, S 130-132 
4. Knuth DE (1968) Fundamental algorithms. The art of computer programming, Bd. 1. Addison-Wesley, Reading

5. Lohberg R, Lutz Th (1963) Was denkt sich ein Elektronengehirn? Eine verständliche Einführung in die Arbeitsweise der Elektronenrechner. Franckh'sche Verlagshandlung, Stuttgart

6. Lutz Th (1959) Stochastische Texte. Augenblick 4(1):3-9

7. Nake F (1992) Informatik und die Maschinisierung von Kopfarbeit. In: Coy W et al (Hrsg) Sichtweisen der Informatik. Vieweg, Braunschweig, Wiesbaden, S 181-204

8. Naur P (1960) Report on the Algorithmic Language ALGOL 60. Commun ACM 3(5):299-314

9. Perlis AJ (1966) A new policy for algorithms? Commun ACM $9(6): 255$ 